\title{
FAILURE OF COMPOSITES WITH SHORT FIBERS
}

Strength-based failure criteria are commonly used with the finite element method (FEM) to predict failure events in composite structures. The laminate analogy is very useful for the calculation of the strength of composite materials with short fibers. The prediction of the laminate strength is carried out by evaluating the stress state within each layer of the laminate based on the classical lamination theory. In this paper FEM is used as a tool to predict the laminate strength. Failure criteria are used to calculate a failure index (FI) from the computed stresses and user-supplied material strengths. The micromechanical analysis has been carried out using computer package MATLAB and numerical simulation has been executed by using a commercially available ANSYS code.

Keywords: composite structures, short fibers -reinforced composites, failure criteria, failure index

\section{Introduction}

Specifically fiber-reinforced composites are one of the most widely used man-made composite materials; they are constituted by reinforcing fibers embedded in a matrix material.

The strength of the uniaxially oriented fibrous structures strongly depends on the length of reinforcing fibers in case of short fibers composites. The fiber and matrix properties, fiber aspect ratio and volume fraction as well as their orientation distribution strongly influence the mechanical response of these materials. Due to the complexity of their microstructure, damage in short fiber composites is extremely difficult to assess numerically or experimentally. Damage involves several types of local degradation processes such as matrix microcracking, fiber/matrix debonding leading to fiber pullout and breakage, etc. These damage mechanisms can occur successively or simultaneously and thus reduce the overall properties of the composite.

Modelling can play an important role in the analysis and design of fiber-reinforced composite materials. Their mechanical properties and possible failure modes can be predicted early during the design stage using effective modeling techniques such, as FEM, boundary element method, a fast boundary element method, meshfree and meshless method, etc. [6, 8]. Recent developments in commercial FEA packages allow the designer to make the detailed analysis of composites.

In a laminate, stresses in the individual layers with different orientations are generally different. Therefore, some of the layers probably reach their limiting stresses before the other remaining layers and they fail first. This is generally referred to as first-ply failure $[1,9]$. A fiber-reinforced laminate may or may not be able to carry loads except during the failure initiation, depending on the nature of the first failure. There are two factors contributing to this behaviour. First, the constituent materials are brittle in nature and do not tolerate local failures. The second factor is a large difference in stiffness and strength between the two principal material directions in a layer.

This paper pertains to the micromechanical analysis of composite materials with short fibers. The micromechanical analysis takes into account the nature of the constituents and their distribution. It can be used to evaluate the overall properties of composites. Failure criteria are used to calculate a failure index (FI) from the computed stresses and user-supplied material strengths. The micromechanical analysis has been carried out using computer package MATLAB. FEM is used as a tool to predict the laminate strength based on the classical lamination theory [3]. Numerical simulation has been prepared by using a commercially available ANSYS code.

\section{Micromechanics of composite material with short fibers}

The basic building block of a composite structure is a unidirectional continuous fiber-reinforced lamina. Placed at various angles of several unidirectional laminae we get a general composite structure. The classical laminate theory is the most commonly used theory for analysing composites with randomly - oriented short fibers $[7,10]$. The laminates with the orientation of angles

\footnotetext{
* Milan Zmindak ${ }^{1}$, Daniel Riecky ${ }^{1}$, Josef Soukup ${ }^{2}$

${ }^{1}$ Department of Applied Mechanics, Faculty of Mechanical Engineering, University of Zilina, Slovakia, Email: Milan.Zmindak@fstroj.uniza.sk

${ }^{2}$ Department of Machinery and Mechanics, Faculty of Production Technology and Management, Jan Evangelista Purkyne University in Usti nad Labem,
} 
$[0 / \pm 45 / 90]$ and $[0 / \pm 60]$ are particularly very suitable for practical applications. In order to predict the strength of this type of composite, it is best to use the maximum strain criterion and then the strength of a composite with randomly-oriented short fibers can be determined by using the properties of unidirectionally reinforced composites with short fibers.

Transverse shear stresses are important in failure investigation of composite laminates, primarily because they cause delamination. The longitudinal and transverse modules of these composites can be expressed by Halphin Tsai equations [7]

$$
\begin{array}{ll}
E_{1}=E^{(m)} \frac{1+\frac{l}{d} \zeta_{E} \eta_{L} \xi}{1-\eta_{L} \xi} & E_{2}=E^{(m)} \frac{1+\zeta_{E} \eta_{T} \xi}{1-\eta_{T} \xi} \\
G_{12}=G^{(m)} \frac{1+\zeta_{E} \eta_{G} \xi}{1-\eta_{G} \xi} & v_{12}=v^{(m)} \frac{1+\zeta_{E} \eta_{\nu} \xi}{1-\eta_{\nu} \xi}
\end{array}
$$

where

$$
\begin{array}{rr}
\eta_{L}=\frac{\frac{E^{(f)}}{E^{(m)}}-1}{\frac{E^{(f)}}{E^{(m)}}+\zeta_{E} \frac{1}{d}} & \eta_{T}=\frac{\frac{E^{(f)}}{E^{(m)}}-1}{\frac{E^{(f)}}{E^{(m)}}+\zeta_{E}} \\
\eta_{G}=\frac{\frac{G^{(f)}}{G^{(m)}}-1}{\frac{G^{(f)}}{G^{(m)}}+\zeta_{E}} & \eta_{v}=\frac{\frac{v^{(f)}}{v^{(m)}}-1}{\frac{v^{(f)}}{v^{(m)}}+\zeta_{E}}
\end{array}
$$

while the superscripts $(m)$ and $(f)$ refer to matrix and fiber, respectively, $\zeta_{E}$ is a reinforcing factor. It depends on the geometry of the fiber in a composite, the packing arrangement of the fibers and its loading conditions. It ranges in value between 1 and 2 . However, only when a reliable experimental value of the $E_{2}$ is available for a composite, the factor $\zeta_{E}$ can be derived and then applied to predict the $E_{2}$ for a range of fiber-volume ratios of the same composite.

A random microstructure results in transversely isotropic properties on a meso-scale level. A simple alternative is to assume that the random microstructure is well-approximated by a periodic microstructure model (Fig.1) [9]. Periodic microstructure mechanics exploits the geometric periodicity of the system in order to simplify mechanical field variables, such as stress, strain, and stiffness. In general, there is a correlation between all of these terms and the position inside the representative volume element (RVE) [11].

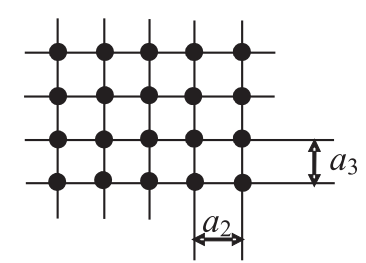

Fig. 1 A periodic microstructure model, square array of fibers.
A simpler alternative is to assume that the random microstructure is well approximated by the hexagonal microstructure displayed in Fig. 2.

The elastic properties of a homogenized material can be computed by [2], i.e. the longitudinal and transversal Young's moduli $E_{1}$ and $E_{2}$, the longitudinal and transversal Poisson's ratios $v_{12}$ and $v_{21}$ and the longitudinal shear modulus $G_{12}$, as follows

$$
\begin{aligned}
& E_{1}=C_{11}-2 C_{12}^{2} /\left(C_{22}+C_{33}\right) \\
& v_{12}=C_{12} /\left(C_{22}+C_{33}\right) \\
& E_{2}=\left(C_{11}\left(C_{22}+C_{33}\right)-2 C_{12}^{2}\right)\left(C_{22}-C_{33}\right) /\left(C_{11} C_{22}-C_{12}^{2}\right) \\
& C_{12}=C_{66}
\end{aligned}
$$

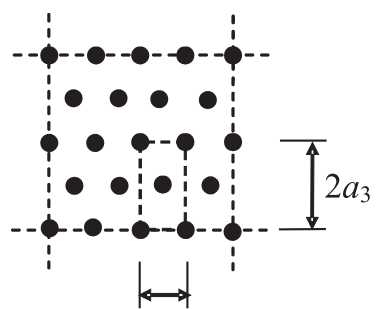

Fig. 2 A hexagonal microstructure model

In order to evaluate the elastic matrix $C$ of a composite, the RVE (fig. 3) is subjected to an average strain.

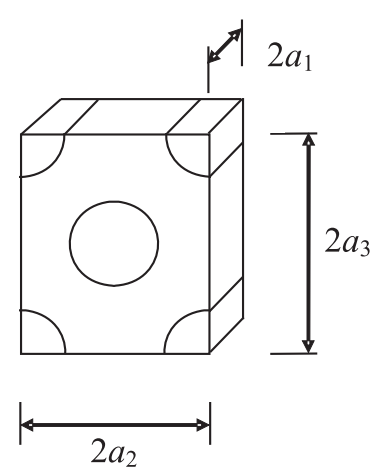

Fig. 3 Representative volume element (RVE)

Then the volume average of the strain in the RVE equals to the applied strain

$$
\bar{\varepsilon}_{i j}=\frac{1}{V} \int_{V} \varepsilon_{i j} d V
$$

The components of the tensor $C$ are determined by solving three elastic models of the RVE with its parameters $\left(a_{1}, a_{2}, a_{3}\right)$ subjected to the boundary conditions $(\mathrm{BC})$. The unit strain applied to the boundary results in a complex state of stress in the RVE. 
Subsequently, the volume average of stress in the RVE equals the required components of the elastic matrix as follows

$$
C_{i j}=\bar{\sigma}_{i}=\frac{1}{V} \int_{V} \sigma_{i} d V .
$$

The coefficients in $C$ are specified by setting a different problem for each column of $C$. Then the components $C_{i j}$ are determined in three steps:

1. For the components $C_{i 1}(i=1,2,3)$, the strain is applied to stretch the RVE in the fiber direction $x_{1}$

$$
\begin{aligned}
& \varepsilon_{1}^{0}=1, \\
& \varepsilon_{2}^{0}=\varepsilon_{3}^{0}=\gamma_{4}^{0}=\gamma_{5}^{0}=\gamma_{6}^{0}=0
\end{aligned}
$$

and the applied $\mathrm{BC}$ are

$$
\begin{aligned}
& u_{1}\left(a_{1}, x_{2}, x_{3}\right)=a_{1}, u_{2}\left(x_{1}, a_{2}, x_{3}\right)=0, u_{3}\left(x_{1}, x_{2}, a_{3}\right)=0, \\
& u_{1}\left(0, x_{2}, x_{3}\right)=0, u_{2}\left(x_{1}, 0, x_{3}\right)=0, u_{3}\left(x_{1}, x_{2}, 0\right)=0,
\end{aligned}
$$

The coefficients $C_{i 1}$ are specified by using the expression

$$
C_{i 1}=\bar{\sigma}_{i} .
$$

2. For the components $C_{i 2}(i=1,2,3)$, the strain is applied to stretch the RVE in the direction $x_{2}$.

$$
\begin{aligned}
& \varepsilon_{2}^{0}=1, \\
& \varepsilon_{1}^{0}=\varepsilon_{3}^{0}=\gamma_{4}^{0}=\gamma_{5}^{0}=\gamma_{6}^{0}=0
\end{aligned}
$$

and the applied $\mathrm{BC}$ are

$$
\begin{aligned}
& u_{1}\left(a_{1}, x_{2}, x_{3}\right)=0, u_{2}\left(x_{1}, a_{2}, x_{3}\right)=a_{2}, u_{3}\left(x_{1}, x_{2}, a_{3}\right)=0, \\
& u_{1}\left(0, x_{2}, x_{3}\right)=0, u_{2}\left(x_{1}, 0, x_{3}\right)=0, u_{3}\left(x_{1}, x_{2}, 0\right)=0,
\end{aligned}
$$

Again, the coefficients $C_{i 2}$ are specified by using

$$
C_{i 2}=\bar{\sigma}_{i} .
$$

3. For the components $C_{i 3}(i=1,2,3)$, the following strain is applied to stretch the RVE in the direction $x_{3}$.

$$
\begin{aligned}
& \varepsilon_{3}^{0}=1, \\
& \varepsilon_{1}^{0}=\varepsilon_{2}^{0}=\gamma_{4}^{0}=\gamma_{5}^{0}=\gamma_{6}^{0}=0
\end{aligned}
$$

and applied $\mathrm{BC}$ are

$$
\begin{aligned}
& u_{1}\left(a_{1}, x_{2}, x_{3}\right)=0, u_{2}\left(x_{1}, a_{2}, x_{3}\right)=0, u_{3}\left(x_{1}, x_{2}, a_{3}\right)=a_{3}, \\
& u_{1}\left(0, x_{2}, x_{3}\right)=0, u_{2}\left(x_{1}, 0, x_{3}\right)=0, u_{3}\left(x_{1}, x_{2}, 0\right)=0,
\end{aligned}
$$

Equally, the coefficients $C_{i 3}$ are specified by using
$C_{i 3}=\bar{\sigma}_{i}$.

The coefficient $C_{44}$ can be determined as follows

$$
C_{44}=\frac{1}{2}\left(C_{22}-C_{33}\right) \text {. }
$$

The fiber-volume fraction is expressed as

$$
v_{f}=\frac{\pi}{\sqrt{3}}\left(\frac{d^{2}}{2 a^{2}}\right) \text {. }
$$

\section{Failure criteria for fiber-reinforced orthotropic layers}

The strength of unidirectional fibers composite materials depends on the direction of fibers on a macroscopic scale. Composite layers are much stronger in the fibers direction than in the direction perpendicular to their fibers. For loads that are primarily parallel to the fibers, either in tension or compression, the material strength is generally determined by the failure of the fibers. For loads transverse to the fibers, failure is controlled by the failure of the much weaker matrix material. The strength of a composite layer in any other direction is based on various failure criteria [4]. Failure criteria for unidirectional fiber composites used in this paper assume a state of plane stress and are therefore only applicable to thin laminates. New 3D criteria for thick composites are derived in [12].

\subsection{Maximum stress and maximum strain criteria}

The basic assumption in predicting the failure of fiber-reinforced layers using the maximum stress and maximum strain criteria is the same as for any other isotropic material. Failure is assumed when the maximum stress along the fiber or transverse to the fiber directions exceeds the strengths in tension or compression.

The failure surface is defined as

$$
\begin{array}{lll}
\sigma_{1}<X_{t}, \quad \sigma_{2}<Y_{t} & \text { for } & \sigma_{1}, \sigma_{2}>0 \\
\sigma_{1}>-X_{c}, \quad \sigma_{2}>-Y_{c} & \text { for } & \sigma_{1}, \sigma_{2}<0 \\
\left|\tau_{12}\right|<S & &
\end{array}
$$

where $X$ and $Y$ represent the ultimate strengths along and transverse to the fiber directions respectively, indexes ${ }_{t}$ and ${ }_{c}$ refer to tension and compression, respectively, $S$ is the ultimate in-plane shear strength of a specimen under pure shear loading.

Similarly, the maximum strain criterion states that failure occurs when one of the following inequalities is violated

$$
\begin{aligned}
& \varepsilon_{1}<\frac{X_{t}}{E_{1}}, \quad \varepsilon_{2}<\frac{Y_{t}}{E_{2}}, \quad \varepsilon_{1}, \varepsilon_{2}>0 \\
& \varepsilon_{1}>-\frac{X_{c}}{E_{1}}, \quad \varepsilon_{2}>-\frac{Y_{c}}{E_{2}}, \quad \varepsilon_{1}, \varepsilon_{2}<0
\end{aligned}
$$


$\left|\gamma_{12}\right|<\frac{S}{G_{12}}$

\subsection{Tsai-Wu criterion}

A more general form of the failure criterion for orthotropic materials under plane stress is expressed as [7]

$$
\begin{aligned}
& F_{01} \sigma_{1}+F_{11} \sigma_{1}^{2}+2 F_{12} \sigma_{1} \sigma_{2}+F_{02} \sigma_{2}+ \\
& +F_{22} \sigma_{2}^{2}+F_{44} \tau_{12}^{2}<1
\end{aligned}
$$

where

$$
\begin{aligned}
& F_{01}=\frac{1}{X_{t}}-\frac{1}{X_{c}}, F_{11}=\frac{1}{X_{t} X_{c}}, F_{02}=\frac{1}{Y_{t}}-\frac{1}{Y_{c}}, F_{22}=\frac{1}{Y_{t} Y_{c}}, \\
& F_{12}=-\frac{1}{2} \frac{1}{\sqrt{X_{t} X_{c} Y_{t} Y_{c}}}, F_{44}=\frac{1}{S^{2}} .
\end{aligned}
$$

The failure criterion for an orthotropic material under a strain is expressed as

$$
\begin{aligned}
& G_{01} \varepsilon_{1}+G_{11} \varepsilon_{1}^{2}+G_{12} \varepsilon_{1} \varepsilon_{2}+G_{02} \varepsilon_{2}+ \\
& +G_{22} \varepsilon_{2}^{2}+G_{44} \gamma_{12}^{2}<1
\end{aligned}
$$

where

$$
\begin{aligned}
& G_{01}=F_{01} E_{11}+F_{02} E_{12}, G_{02}=F_{02} E_{22}+F_{01} E_{12}, \\
& G_{11}=F_{11} E_{11}^{2}+F_{22} E_{12}^{2}+F_{12} E_{11} E_{12}, \\
& G_{22}=F_{22} E_{22}^{2}+F_{11} E_{12}^{2}+F_{12} E_{22} E_{12}, \\
& G_{12}=2 E_{12}\left(F_{11} E_{11}+F_{22} E_{22}\right)+2 F_{1}\left(E_{12}^{2}+E_{11} E_{22}\right), \\
& G_{44}=F_{44} E_{44}^{2} .
\end{aligned}
$$

When $F_{12}=\frac{-1}{2 X_{t}^{2}}$, the Tsai-Wu criterion is reduced to the Tsai-Hill criterion, and when $F_{12}=\frac{-1}{2 X_{t} X_{c}}$ the Tsai-Wu criterion is reduced to the Hoffman criterion [7].

These failure criteria are used to calculate a failure index (FI) from the computed stresses and user-supplied material strengths. The failure index as a response of quantity is used for several FEM packages and it is defined as

$$
I_{F}=\frac{\text { stress }}{\text { strenght }} .
$$

Failure criteria predict the first occurrence of failure in one of the laminate layers. A value less than 1 denotes no failure and failure is predicted when $I_{F} \geq 1$. The strength ratio is the inverse of the failure index.

It is important to distinguish between the fiber failure (FF) and the inter-fiber failure (IFF). In the case of shear plane stress, the IFF criteria discriminates three different modes [1]. The IFF Mode $A$ is when perpendicular transversal cracks appear in the lamina under transverse tensile stress with or without in-plane shear stress. The IFF Mode $B$ denotes perpendicular transversal cracks, but in this case they appear under in-plane shear stress with small transverse compression stress. The IFF Mode $C$ indicates the start of oblique cracks when the material is under significant transversal compression.

The FF and the three IFF modes yield separate failure indices. The failure index for FF is defined as

$$
I_{F F}=\left\{\begin{array}{lll}
\sigma_{1} / X_{t} & \text { if } & \sigma_{1}>0 \\
\sigma_{1} / X_{c} & \text { if } & \sigma_{1}<0
\end{array} .\right.
$$

For IFF with positive transverse stress, Mode A is active. The failure index in this case is defined as

$$
\begin{aligned}
I_{I F F, A} & =\sqrt{\left(\frac{\tau_{12}}{S}\right)^{2}+\left(1-p_{6 t} \frac{Y_{t}}{S}\right)^{2}\left(\frac{\sigma_{2}}{Y_{t}}\right)^{2}}+p_{6 t} \frac{\sigma_{2}}{S} \\
\text { if } \sigma_{2} & \geq 0, \\
\text { where } p_{6 t} & =0.3 .
\end{aligned}
$$

Under negative transverse stress, either Mode $B$ or Mode $C$ is active, depending on the relationship between in-plane shear stress and transversal shear stress. The failure indices are defined as

$$
\begin{gathered}
I_{I F F, B}=\frac{1}{S}\left[\sqrt{\tau_{12}^{2}+\left(p_{6 c} \sigma_{2}\right)^{2}}+p_{6 c} \sigma_{2}\right], \text { if }\left\{\begin{array}{c}
\sigma_{2}<0 \\
\left|\frac{\sigma_{2}}{\tau_{12}}\right| \leq \frac{F_{2 A}}{F_{6 A}}
\end{array}\right. \\
I_{I F F, C}=-\frac{Y_{c}}{\sigma_{2}}\left[\sqrt{\left(\frac{\tau_{12}^{2}}{2\left(1+p_{2 c}\right) S}\right)^{2}+\left(\frac{Y_{c}}{\sigma_{2}}\right)^{2}}\right], \text { if }\left\{\begin{array}{c}
\sigma_{2}<0 \\
\left|\frac{\sigma_{2}}{\tau_{12}}\right| \geq \frac{F_{2 A}}{F_{6 A}}
\end{array}\right.
\end{gathered}
$$

where $p_{6 c}=0.2$

The limit between Mode $B$ and Mode $C$ is defined by the relation $F_{2 A} / F_{6 A}$, where

$$
\begin{aligned}
& F_{2 A}=\frac{S}{2 p_{6 c}}\left[\sqrt{1+2 p_{6 c} \frac{Y_{c}}{S}}-1\right], \\
& F_{6 A}=S \sqrt{1+2 p_{2 c}}, p_{2 c}=p_{6 c} \frac{F_{2 A}}{S} .
\end{aligned}
$$

\section{Numerical examples}

Example 1:

In this example, a composite with randomly oriented fibers is assumed with its material characteristics for the fibers: $E_{f}=210 \mathrm{GPa}$, 
$v_{f}=0.3$ and for the matrix $E_{m}=31 \mathrm{GPa}, v_{m}=0.15$. The geometrical characteristics of the fibers are $L=6 \mathrm{~cm}, d=0.75 \mathrm{~mm}$.

The material characteristics of the composite material given for a variable amount of fibers are shown in Tab. 1, including their Young's modulus $E$ and Poisson's ratio

Material characteristics

Tab.1

\begin{tabular}{|c|c|c|c|c|c|c|}
\hline & \multicolumn{2}{|c|}{ Halphin Tsai } & \multicolumn{2}{c|}{$\begin{array}{c}\text { Periodic micro- } \\
\text { structure model }\end{array}$} & \multicolumn{2}{c|}{$\begin{array}{c}\text { Classical } \\
\text { laminate theory }\end{array}$} \\
\cline { 2 - 7 } & $\begin{array}{c}30 \\
\mathrm{~kg} / \mathrm{m}^{3}\end{array}$ & $\begin{array}{c}60 \\
\mathrm{~kg} / \mathrm{m}^{3}\end{array}$ & $\begin{array}{c}30 \\
\mathrm{~kg} / \mathrm{m}^{3}\end{array}$ & $\begin{array}{c}60 \\
\mathrm{~kg} / \mathrm{m}^{3}\end{array}$ & $\begin{array}{c}30 \\
\mathrm{~kg} / \mathrm{m}^{3}\end{array}$ & $\begin{array}{c}60 \\
\mathrm{~kg} / \mathrm{m}^{3}\end{array}$ \\
\hline $\begin{array}{c}\text { E Young's } \\
\text { modulus [GPa] }\end{array}$ & 31.644 & 31.795 & 31.239 & 31.426 & 331.470 & 31.960 \\
\hline Poisson's ratio & 0.151 & 0.151 & 0.150 & 0.151 & 0.1470 & 0.1480 \\
\hline
\end{tabular}

For the given amount of fibers of $30 \mathrm{~kg} / \mathrm{m}^{3}$ and $60 \mathrm{~kg} / \mathrm{m}^{3}$, there are only minor differences in the material characteristics regardless of the method used, whether the Halphin Tsai method, the periodic microstructure model or the classical laminate theory. The correlation between the modulus of elasticity and the fibers volume fraction is given in Fig. 4.

The modules of elasticity - the first-ply $E_{1 F A I L}$, the second-ply $E_{2 F A I L}$ and the ultimate failure $E_{3 F A I L}$ in a fictitious laminate [0/45/-45/90]S are used instead of these in a composite with short fibers. The values calculated using the classical laminate theory are given in Tab. 2.

Modules of elasticity

Tab. 2

\begin{tabular}{|c|c|c|}
\hline & $30 \mathrm{~kg} / \mathrm{m}^{3}$ & $60 \mathrm{~kg} / \mathrm{m}^{3}$ \\
\hline$E_{1 \text { FAIL }}[\mathrm{GPa}]$ & 31.47 & 31.96 \\
\hline$E_{2 \text { FAIL }}[\mathrm{GPa}]$ & 23.66 & 24.10 \\
\hline$E_{3 \text { FAIL }}[\mathrm{GPa}]$ & 15.75 & 16.02 \\
\hline
\end{tabular}

The main characteristic essentially changed is the strength of a composite with short fibers. In the absence of more accurate information, the average and characteristic value of an equivalent flexural tensile strength for steel wire fibers can be calculated as follows [5]

$$
R_{e_{m, 150}}=\frac{180 W_{f} \lambda_{f} d_{f}^{1 / 3}}{180 C+W_{f} \lambda_{f} d_{f}^{1 / 3}}
$$

with $C=20$ for hooked-end steel fibers under the trade name of Dramix, where $W_{f}$ is the fiber content (in $\mathrm{kg} / \mathrm{m}^{3}$ ), $d_{f}$ is the diameter of steel fibers, and $\lambda_{f}$ is the ratio between the length and the diameter of steel fibers. The flexural tensile strength $f_{\text {fctm,eq }}$ and characteristic flexural tensile strength $f_{\text {fctk,eq }}$ for fibers are given as (Tab. 3)

$$
f_{\text {fctm }, e q}=\frac{R_{e_{m, 150}} f_{\text {fctm }, e q}}{100}, f_{\text {fctk }, e q}=0.7 f_{\text {fctm }, e q} .
$$

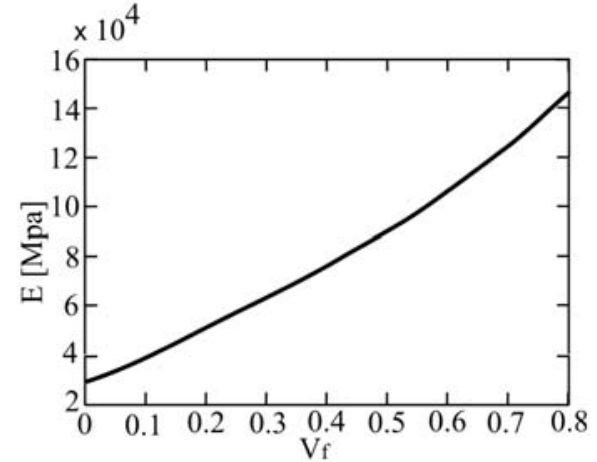

Fig.4. The correlation between the modulus of elasticity and the fiber volume fraction

Flexural tensile strengths

Tab. 3

\begin{tabular}{|c|c|c|c|c|}
\hline & $30 \mathrm{~kg} / \mathrm{m}^{3}$ & $\begin{array}{c}30 \mathrm{~kg} / \mathrm{m}^{3} \\
\mathrm{RC} 80 / 60-\mathrm{BN}\end{array}$ & $60 \mathrm{~kg} / \mathrm{m}^{3}$ & $\begin{array}{c}60 \mathrm{~kg} / \mathrm{m}^{3} \\
\mathrm{ZC} 30 / .50\end{array}$ \\
\hline$f_{\text {fctm,eq }}[\mathrm{MPa}]$ & 2.448 & 2.26 & 3.519 & 3.40 \\
\hline$f_{\text {fctk,eq }}[\mathrm{MPa}]$ & 1.714 & 1.83 & 2.463 & 2.40 \\
\hline
\end{tabular}

Example 2:

In this example, failure indices were determined. In this case the laminate consists of four layers [30/60/90/0] made from the uniform material AS4D/9110 [11] with the uniform thickness. The dimensions and the boundary conditions are described in Fig. 5 .

The model is obtained by using the ANSYS input command sequence and the element SHELL99. Following the generation of model material strengths, the failure indices were calculated. The strength constants of AS4D/9110 material are: $F_{1 t}=1830 \mathrm{MPa}$, $F_{1 c}=-1096 \mathrm{MPa}, F_{2 t}=57 \mathrm{MPa}, F_{2 c}=-228 \mathrm{MPa}, c_{6}=-1$, $c_{5}=-1, c_{4}=-1$.

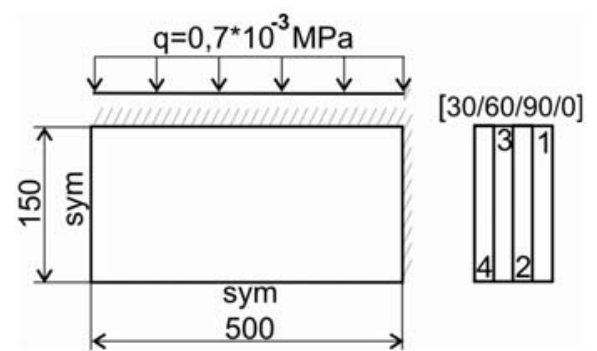

Fig. 5 Loads and the geometry of the laminate and the numbering of its layers

The results are summarized in Tab.4, where $I_{F, M A X} S$ is the maximum stress failure index and $I_{F, T W}$ is the Tsai-Wu failure index. As can be seen from the given results, the maximum FI's are in layer 1. The distribution of failure indices is given in Fig. 9. In the following two layers the maximum FI's occurred in the 


\section{COMMNICOIIONS}

Failure indices

Tab. 4

\begin{tabular}{|c|c|c|c|c|c|c|c|c|}
\hline \multirow{2}{*}{ No. Lamina } & \multicolumn{4}{|c|}{$I_{F, M A X S}$} & \multicolumn{4}{|c|}{$I_{F, T W}$} \\
\hline & Max. & Node & Min. & Node & Max & Node & Min. & Node \\
\hline 1. & $1.29 \mathrm{e}-2$ & 1 & $3.32 \mathrm{e}-7$ & 212 & $9.52 \mathrm{e}-3$ & 519 & $-5.64 \mathrm{e}-5$ & 202 \\
\hline 2. & $7.64 \mathrm{e}-3$ & 4 & $1.23 \mathrm{e}-7$ & 212 & $5.79 \mathrm{e}-3$ & 4 & $-3.63 e-5$ & 202 \\
\hline 3. & $3.56 \mathrm{e}-3$ & 1 & $0.50 \mathrm{e}-7$ & 262 & $1.33 \mathrm{e}-3$ & 4 & $-1.92 \mathrm{e}-4$ & 24 \\
\hline 4. & $3.22 \mathrm{e}-6$ & 202 & $0.35 \mathrm{e}-9$ & 6627 & $0.1 \mathrm{e}-12$ & 202 & $0.34 \mathrm{e}-18$ & 467 \\
\hline
\end{tabular}

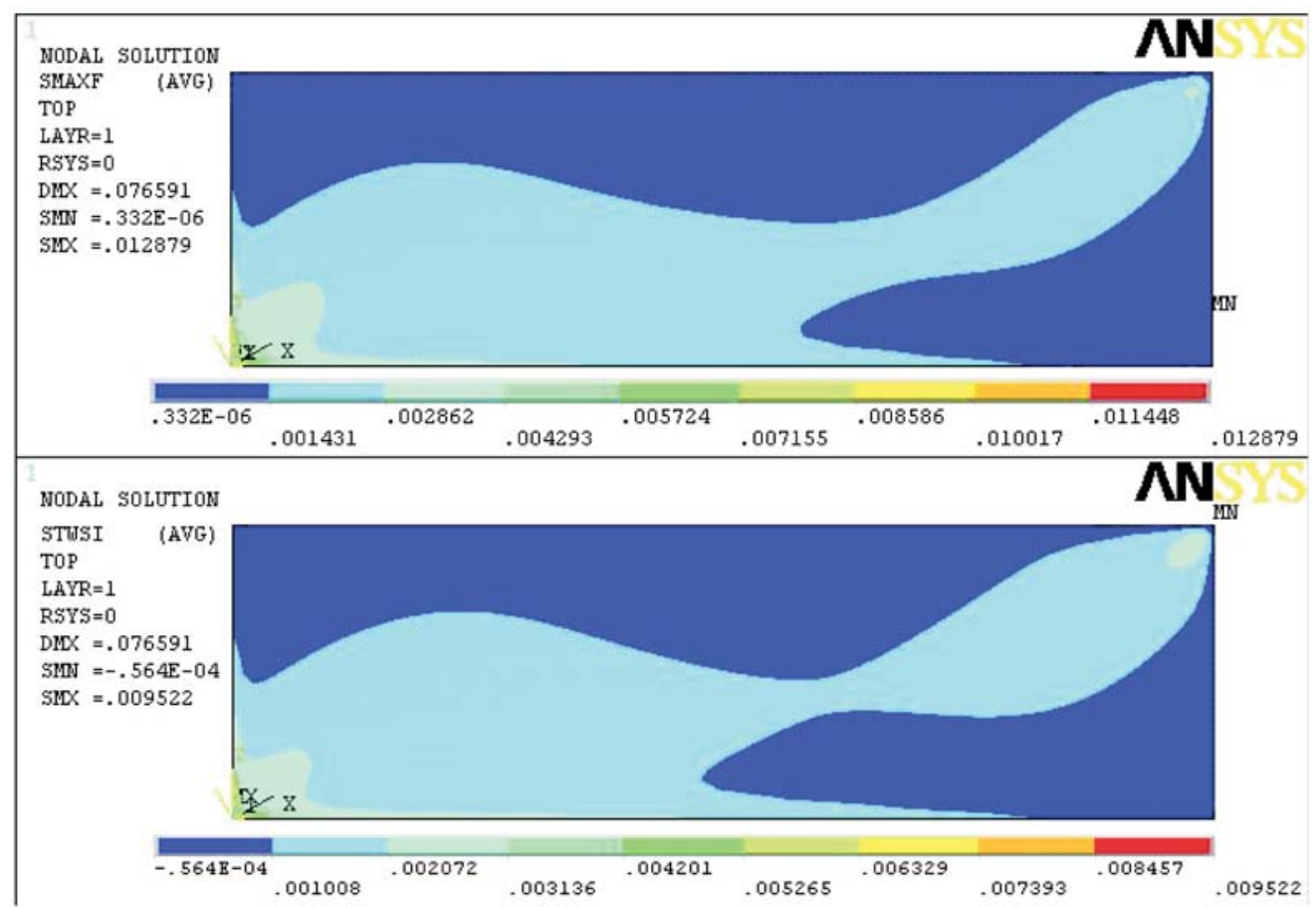

Fig. 9 The distribution of failure indices $I_{F, M A X}, I_{F, T W}$ in the first lamina

middle of the laminate. Moreover, the distribution of the FI's in layer 4 is different, the FI values are the smallest and their locations changed. The minimum values occurred in the corner of the laminate where it is fixed.

\section{Conclusion}

Some calculation methods for the calculation of the modulus of elasticity and the strength of randomly reinforced composite materials are derived in the first example. There are no significant differences in the modulus of elasticity when using the Halphin Tsai method, the periodic microstructure model or the classical laminate theory. The main characteristic that changed essentially is the strength of a composite with short fibers. In the second example failure criteria are presented using failure index. The maximum failure index was calculated using the maximum stress criterion. A laminate plate from AS4D/9110 material was used and the maximum stress failure index $I_{F, M A X S}$ and the Tsai-Wu failure index $I_{F, T W}$ were computed.

\section{Acknowledgements}

The authors gratefully acknowledge the support by the Slovak Science and Technology Assistance Agency registered under number APVV-0169-07, the Slovak Grant Agency VEGA 1/0657/09, and the Czech Science Foundation (Project-No GA CR 101/07/0946). 


\section{References}

[1] ALTENBACH, H., ALTENBACH, J., KISSING. W.: Structural Analysis of Laminate and Sandwich Beams and Plates. Lublin, 2001.

[2] BARBERO, E. J.: Finite Element Analysis of Composite Materials. In: CRC Press : Taylor \& Francis Group, 2008.

[3] CARRERA, E.: Theories and Finite Elements for Multi-layered Plates and Shells. In: Arch. Comput. Meth. Engng. 10 (3), 2003 , pp. 215-296.

[4] DAVILA, C. G., CAMANHO, P. P., ROSE, CH. A.: Failure Criteria for FRP Laminates. In: J. of Composite Materials, Vol. 39, No. 4, 2005, pp. 323-345.

[5] DRAMIX Guideline: Design of Concrete Structures. No. 4, 1995.

[6] KOMPIS, V., ZMINDAK, M., MURCINKOVA, Z.: Trefftz Radial Basis Functions. Proc. of Leuven Symposium on Applied Mechanics in Engineering (LSAME.08) : Part I : Proc. of Trefftz 08, 5th International Workshop on Trefftz Methods, Leuven, 2008.

[7] LAS, V.: Mechanics of Composite Materials (in Czech), West Bohemia University, 2008.

[8] LIU, Y. J., NASHIMURA, N., OTANI, Y., TAKAHASHI, T., CHEN, X. L., MUNAKATA, H.: A Fast Boundary Element Method for Analysis of Fiber - Reinforced Composites Based on a Rigid-Inclusion Model, In: Journal of Applied Mechanics, Vol.72, 2005, pp.115-128.

[9] LUCIANO, R., BARBERO, E. J.: Formulas for the Stiffness of Composites with Periodic Microstructure. In: Int. J. of Solids and Structures, 31 (21), 1995, pp. 2933-2944.

[10] MALLICK, P. K.: Fiber-Reinforced Composites: Materials. In: Manufacturing and Design, CRC Press, Taylor \& Francis, 2007.

[11] QU, J., CHEREKAOIU, M.: Fundamentals of Micromechanics of Solids. John Wiley \& Sons : Hoboken ; New Jersey, 2006.

[12] STAMBLEWSKI, CH., SANKAR, B.V., ZENKERT, D.: Analysis of Three-Dimensional Quadratic Failure Criteria for Thick Composites using the Direct Micromechanics Method, In: Journal of Composite Materials, Vol. 42, No. 7, 2008. 